Proceedings of ASME Turbo Expo 2010: Power for Land, Sea and Air IGTI 2010

June 14-18, 2010, Glasgow, UK

GT2010-22306

\title{
UNSTRUCTURED LARGE EDDY SIMULATION TECHNOLOGY FOR PREDICTION AND CONTROL OF JET NOISE
}

\author{
Yaser Khalighi, Frank Ham, Parviz Moin \\ CASCADE Technologies Inc. \\ Palo Alto, CA 94303
}

\author{
Tim Colonius \\ Dept. Mechanical Engineering \\ California Institute of Technology \\ Pasadena, CA 91125
}

\author{
Sanjiva K. Lele \\ Dept. Mechanical Engineering \\ Stanford University \\ Stanford, CA 94305
}

\section{Introduction}

Development of concepts for reduction of jet noise has relied heavily on expensive experimental testing of various nozzle designs. For example, the design of nozzle serrations (chevron) and internal mixer/ejector nozzles have relied largely on laboratory and full-scale testing. Without a deeper understanding of the sources of high-speed jet noise it is very difficult to effectively design configurations that reduce the noise and maintain other performance metrics such as nozzle thrust. In addition, the high complexity of the flow limits the success of a parametric black-box optimization.

It is our premise that significant new advances in the understanding of noise generation mechanisms for jets and realistic methods for reducing this noise can be developed by exploiting high-fidelity computational fluid dynamics: namely large eddy simulation (LES). In LES, the important energy-containing structures in the flow are resolved explicitly, resulting in a timedependent, three-dimensional realization of the turbulent flow. In the context of LES, the unsteady flow occurring in the jet plume (and its associated sound) can be accurately predicted without resort to adjustable empirical models. In such a framework, the nozzle geometry can be included to directly influence the turbulent flow including its coherent and fine-scale motions. The effects of propulsion system design choices and issues of integration with the airframe can also be logically addressed.

Before discussing the details of LES as it applies to supersonic jet noise prediction, it is important to summarize some of the key LES developments that have led to the successful prediction of many other turbulent flows. This is particularly important as the rapid increase in computational power of the last decade has made time-dependent flow simulations of all sorts ubiquitous. In our opinion, two essential elements of LES are:

1. low dissipation numerical methods, and

2. the use of explicit sub-grid scale models with dynamic closures

The literature on non-dissipative (sometimes called kinetic energy conserving) numerical methods for LES is substantial. In an important comparative study, Mittal \& Moin (1997) illustrated the detrimental effect of upwind-biased schemes on resolved turbulence. Based on this and other similar studies, non-dissipative numerical methods can be shown to much more effectively capture the broad spectral content of turbulence. This is an attribute which is very important for noise prediction. Dynamic method for closing the sub-grid scale models based on the resolved flow was first proposed by Germano et al. (1991) and modified by Lilly (1992). Use of the dynamic method was extended to com- 
pressible flows by Moin et al. (1991) and to one-equation models by Ghosal et al. (1995). More recently, the dynamic method has been applied to Vreman's model Vreman (2004); You \& Moin (2007). Implementing the dynamic method in the context of nondissipative schemes results in a numerical approach with no tunable turbulence parameters, apart from the details of the mesh.

Following this pioneering work, combination of nondissipative numerical schemes and dynamic models has been applied to predict an increasingly complex array of flows and their sound field accurately. Wang et al. (2006) reviewed recent advancement of LES in computational aeroacoustics. In earlier attempts, Wang \& Moin (2000) applied incompressible LES and acoustics analogy for computing the sound emitted from trailing edges. This framework was later utilized by Marsden et al. (2007) to optimize the shape of a trailing edge for noise mitigation. As these methods of noise prediction are based on structured flow solvers for modeling noise sources and approximate Green's functions for propagation/scattering of sound sources, their predictive capability is limited to the flow-generated sound induced by fairly simple geometries. Recently, Khalighi et al. (2010) developed a technique for predicting the sound generated by low-Mach number flows in the presence of arbitrarilycomplex geometries by using unstructured, incompressible LES solver of Ham et al. (2007) in conjunction with a boundary element method.

It should be noted that different groups who have attempted to predict the noise of turbulent jets using LES have had mixed success (see Ladeinde et al. (2008); Lo et al. (2008)). As reviewed by Bodony \& Lele (2008), numerical dissipation, inadequate azimuthal resolution, and artificially thick near-nozzle shear layers were among the factors which contributed to poor predictions. In the work by Shur et al. $(2005 a, b)$; Spalart et al. (2007), a high-order, multi-block structured LES code was successfully used for predicting the jet noise. Despite the accurate prediction, due to the structured nature of the algorithm, the interaction of nozzle and flow was not directly included. The application of the method was, therefore, limited to simple nozzle design. To extend the use of LES as a design tool for jet noise reduction, since the nozzle geometry directly influences the turbulent flow inducing coherent and fine-scale motions, an unstructured mesh framework should be considered. The effect of propulsion system design choices including integration with the airframe can be methodically, and effectively addressed within such a framework. Most recently, Mendez et al. (2009, 2010) have successfully applied unstructured LES scheme of Shoeybi et al. (2009) and predicted the noise emitted from supersonic jets and compared the result to the experiments carried out by Bridges \& Wernet (2008). An important lesson learned from previous research on computational aeroacousitcs is that a numerical method suitable for accurate prediction of noise requires extra care in many different aspects. In general, sound waves contain a small amount of energy as compared to the flow it- self and can be easily overwhelmed by numerical errors. For the same reason, sponge layers are required to prevent reflection of sound from outflow boundary conditions back to the computational domain (Bodony, 2005). Even, shock capturing schemes can severely attenuate sound waves if not applied properly (Mani et al., 2009).

Based on the experience gained in the mentioned research, a novel numerical scheme for unstructured compressible LES which is less sensitive to the quality of the grid has been developed. This numerical scheme is the core of the unstructured LES technology used in the present work. This technology (briefly introduced in §2) is targeted for performing large-scale, high-fidelity simulations of turbulent flows in complex configurations. In $\S 3$ this technique was utilized to predict the flow and noise emitted from supersonic jets. In this work, perfectly expanded unheated and heated supersonic jets from a round nozzle were considered. The nozzle geometry and operating conditions matched those of the test cases previously conducted at UTRC Acoustics Research Tunnel (ART) (see Schlinker et al. (2008)). Despite the similarity in the physics of flow, the main difference of current work to the most recent work of Mendez et al. (2009, 2010) is:

1. Due to the accuracy of the numerical scheme, a more aggressive grid refinement was used here to improve the local resolution in the vicinity of the nozzle lips and

2. Comparisons to experiment were conducted in a blind fashion. LES predicted flow field, near field hydrodynamic pressures, and farfield noise were sent to UTRC for comparison with measurements conducted in the ART. The jet hydrodynamic near field predictions were motivated by UTRCs prior demonstration of the aft radiated jet noise being controlled by shear layer instability wavepackets (see Reba et al. $(2008,2009))$. These wavepackets correspond to the large scale turbulence structures identified by many researchers and considered to be "source" responsible for noise radiating to the far field in the aft direction. The structures, measured by UTRC, are the input to a convected wavepacket model that successfully linked the near field source with acoustic far field. The current study was designed to predict spectra at the same near field and far field stations as measured by UTRC to provide validation of the computational capability for predicting the organized structure characteristics. Fine scale mixing noise is also embedded in the current LES methodology.

Comparison of LES results with UTRC experiments and discussions will be presented in $\S 4$. $\S 5$ concludes this paper by summarizing the work, describing ongoing efforts for improving the results and suggesting future directions. 


\section{Unstructured LES Technology}

The LES software used here is composed of pre-processing tools (i.e. mesh generation), a flow solver "CharLES", and postprocessing tools. The mesh generation module produces highquality yet economical unstructured grids suitable for capturing turbulence dynamics. The flow solver utilizes a low-dissipative numerical scheme designed to produce accurate results on unstructured meshes, in particular in the presence of hanging nodes and other transition type elements. The database generated by LES is then processed by the post-processing module for statistical analysis of flow and noise as well as flow visualization. These modules are briefly described in the following subsections.

\subsection{Mesh generation and pre-processing}

Performing a careful LES computation requires a highquality mesh, with adequate resolution applied in the regions of interest. An unstructured grid can easily be locally refined or coarsened as appropriate to capture the flow features. Transitional type elements ( tetras, and pyramids) as well as polygonal type elements (hanging nodes) can easily be produced to accomplish this . In the simulation of turbulent jets to be presented, a high resolution grid is needed in the vicinity of the nozzle where small-scale structures are present due to the transition of a thin shear layer to turbulence. Other parts of the flow can easily be captured with a less resolved grid.

For this reason, we have developed algorithms to generate high quality grids with azimuthal zonal refinement by introducing transitional elements. The motivation for devising this algorithm was that in previous published LES attempts, the azimuthal resolution of the mesh was far less than the axial and radial resolution, in particular in the vicinity of the nozzle lip (see Mendez et al. (2010); Shur et al. (2005a,b); Spalart et al. (2007)).

Figure 1 demonstrates an LES grid generated for the nozzle geometry of interest. In this grid the number of azimuthal grid points in various zones as well as slices normal to the axis of the jet is shown. Progressively higher resolution in the vicinity of the nozzle lip is considered. Farther down-stream of the jet, scales of turbulence increase and less mesh density is required. Zonal mesh refinement algorithm, together with unstructured mesh capability of our LES software allows us to perform high-resolution yet cost-effective jet simulations.

\subsection{CharLES: unstructured LES for compressible flows}

The large eddy simulations described in this report were performed with the flow solver "CharLES". CharLES solves the spatially-filtered compressible Navier-Stokes equations on unstructured grids using a novel control-volume based finite volume method where the flux is computed at each control volume face using a blend of a non-dissipative central flux and a dissipative upwind flux, i.e.:

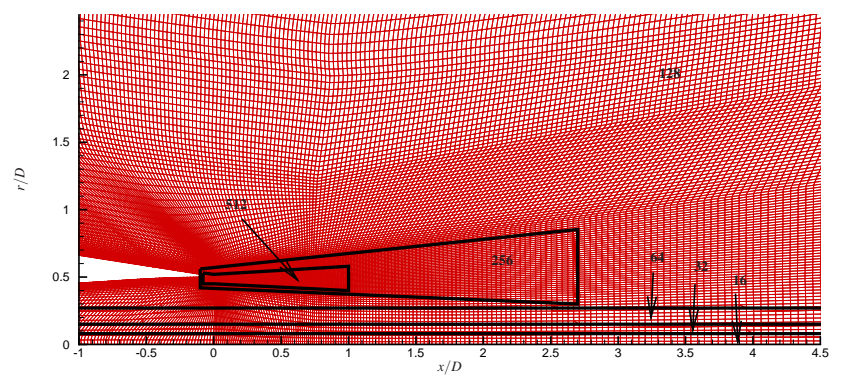

(a) Azimuthal refinement levels

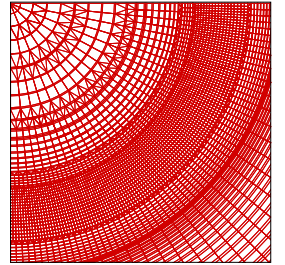

(b) $x / D=0.5$

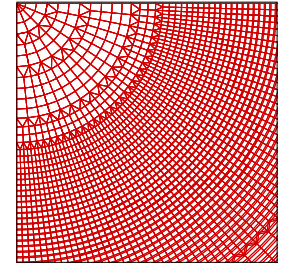

(c) $x / D=2$

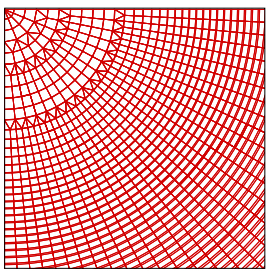

(d) $x / D=3$
Figure 1. Grid with zonal refinement generated for jet simulations.

$$
F=(1-\alpha) F_{\text {central }}+\alpha F_{\text {upwind }}
$$

where $0 \leq \alpha \leq 1$ is a blending parameter. This blending approach is often the basis of implicit approaches to LES, where the blending parameter is selected as a global constant with a value large enough to provide all the necessary dissipation (and potentially much more). For example, in the turbulent jet literature Tucker (2004) used this approach and reported that the smallest "usable" value of blending parameter was determined to be $\alpha=0.25$. The treatment is described in detail by Shur et al. (2003). In later work, Xia et al. (2009) reported that the minimum value of blending parameter was set to 0.1 "to avoid numerical instability".

CharLES does not use the "implicit" LES approach - an explicit sub-grid scale model is used to model the effect of the unresolved scales on the resolved flow (see $\S 2.3$ below for details). To minimize numerical dissipation relative to implicit LES approaches, the value of $\alpha$ is allowed to vary spatially such that it can be set to zero in regions where the grid quality is good and the scheme based on the central flux is discretely stable and non-dissipative. In regions of less-than-perfect grid quality, however, the central scheme can introduce numerical instabilities that must be prevented from contaminating/destabilizing the solution by locally increasing $\alpha$. The novel aspect of CharLES is its algorithm to compute this locally optimal (i.e. minimal) $\alpha$, which will be described next. 

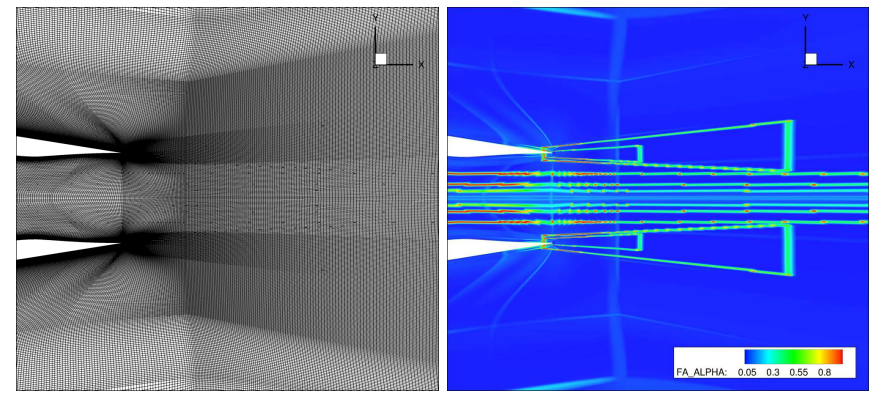

Figure 2. Left) section through centerline of medium jet mesh; right) computed local blending parameter based on $\alpha=c\left\|\left(D_{n}+D_{n}^{T}\right)_{i}\right\|$ with $c=2$.

2.2.1 $\alpha$ heuristic A stable and non-dissipative differencing operator is a skew-symmetric operator, i.e. $D=-D^{T}$. If one constructs a differencing operator on a uniform Cartesian grid using polynomial interpolation, one produces a skewsymmetric operator naturally. On non-uniform and/or irregular grids, however, the application of polynomial interpolation to build accurate face fluxes will lead to a non-skew-symmetric differencing operator. It is this local lack of skew-symmetry that CharLES uses to scale the blending parameter $\alpha$. Specifically, we use the row-norm of the symmetric part of the differencing operator $D$ :

$$
\alpha=c\left\|\left(D_{n}+D_{n}^{T}\right)_{i}\right\|
$$

where $c=2$ is a constant chosen based on numerical tests. One significant advantage of this approach is that the blending parameter is purely grid-based, and can be pre-computed based on the operators only. Figure 2 illustrates how this approach introduces the dissipation only where required in the region of the grid transitions for the zonal grid shown in figure 1.

\subsection{Sub-grid scale modeling}

Because the underlying numerical method has minimal numerical dissipation, it is critical to employ a sub-grid model to account for the physical effects of the unresolved turbulence on the resolved flow. Two modeling options are available in the code: the dynamic Smagorinsky model (Germano et al., 1991; Lilly, 1992; Moin et al., 1991) and a dynamic version of Vreman's model (Vreman, 2004; You \& Moin, 2007). For the large eddy simulations reported in this work, we used the Vreman model with constant coefficient set to the recommended value of $c=0.07$, and constant turbulent Prandtl number $P r_{t}=0.9$ to close the energy equation.

\subsection{Shock-capturing}

While the shocks that exist in the jet simulations carried out to date are weak, strong shocks will be present for the pressuremismatched conditions and more complex geometries planned for the future. Shocks, like sub-grid scale turbulence, are also sub-grid phenomena and thus require modeling to account for their effect on the resolved flow. Unlike sub-grid scale turbulence they are localized in the flow, and a surgical introduction of modeling is potentially more appropriate. CharLES uses a hybrid Central-WENO scheme to simulate flows involving shocks. The scheme has three pieces:

1. A central scheme, described previously,

2. An scheme appropriate for computing a flux across a shock,

3. A hybrid switch, which detects where shocks are present in the flow, and activates the shock-appropriate scheme.

For the shock-appropriate scheme, CharLES uses a 3rdorder WENO method to perform reconstructions (Shi et al., 2002), and the HLLC approximate Riemann solver to compute the flux (Harten et al., 1983). The WENO method is fully unstructured, and as such must consider a potentially large number of candidate stencils. In regions of nearly uniform orthogonal grids, however, the number of candidate stencils reduces to two stencils per face-side (i.e. two for the left side value and two for the right-side value at each face), substantially reducing the cost of the method over a large fraction of the grid.

The hybrid switch is based on the method developed originally by Hill \& Pullin (2004), where the magnitudes of the smoothness parameters computed as part of the WENO reconstructions are compared to identify the presence of flow discontinuities.

\subsection{Post-processing}

For analysis of data generated by computation and in particular noise calculation, a time record of flow variables is required. In many situations, the locations where recording data is required are not known in advance. As a result, storing the entire volumetric flow-field in time is needed. For the purpose of noise prediction of turbulent flows using LES in a wide range of frequencies, a long data record is required to obtain a converged solution. Consequently, the database generated by such simulations are extremely large. In a medium size LES calculation of a supersonic jet, the size of the database can be as large as $10 \mathrm{~TB}$. To process the large volumes of data, we developed a post-processing module that can efficiently read the snapshots of flow-field, perform spatial and temporal statistical analysis of the data, and visualize the flow field. This module is also applied for computation of noise in the farfield. 


\subsection{Calculation of farfield noise}

Surface projection techniques are widely used for computation of farfield noise. These techniques are analytical methods based on Green's functions corresponding to the wave equation. They relate the sound at a farfield point to velocity and pressure computed (or measured) in the nearfield. The reasons for applying a secondary tool to evaluate sound, which can be obtained from the flow solver itself, is twofold:

1. Direct computation of sound at farfield locations requires the extension of computational domain to farfield. From the computational perspective, this extension is prohibitively expensive.

2. Sound waves carry only a minuscule energy of the flow. Consequently, they can be easily overwhelmed by numerical errors caused by low order numerical schemes used for unstructured flow solver. The effect of numerical errors is minimized by using analytical methods.

Variants of acoustic surface projection techniques are used by different groups. For a review of these methods see the article by Lyrintzis (2003). For prediction of hot supersonic jet noise we developed a noise projection module based on the early work of Ffowcs Williams \& Hawkings (1969) and its extension by Spalart \& Shur (2009). According to the original formulation of Ffowcs Williams \& Hawkings (1969), sound at a farfield location can be computed from flow information on an arbitrarilyshaped surface $(S)$ and the volume-distibuted sources outside of $S$. Due to the difficulties associated with using volumedistributed sources, surface $S$ is often chosen such that it encloses flow-generating sound sources. As a result, the volume term can be assumed small enough and its effect can be neglected. For simulation of hot jets, Spalart \& Shur (2009) argues that neglecting the volume term can be erroneous, where surface $S$ fails to entirely enclose the region of turbulence. They demonstrate that a pressure-based variant of the original formulation can reduce this error. This conclusion was revisited and verified in a recent work of Mendez et al. (2010). According to these studies, the pressure formulation is best-suited for noise prediction of hot supersonic jets and is applied in the present work.

Time accurate flow variables are collected on a surface $S$ that encloses the sources of sound (see figure 3 ). The following terms are extracted from the surface $S$ :

$$
\begin{aligned}
& F_{1}=\frac{p^{\prime} n_{i} r_{i}+\left(\rho_{\infty}+p^{\prime} / c_{\infty}^{2}\right) u_{i} u_{j} n_{i} r_{j}}{c_{\infty} r^{2}}+\frac{\left(\rho_{\infty}+p^{\prime} / c_{\infty}^{2}\right) u_{i} n_{i}}{r} \\
& F_{2}=\frac{p^{\prime} n_{i} r_{i}+\left(\rho_{\infty}+p^{\prime} / c_{\infty}^{2}\right) u_{i} u_{j} n_{i} r_{j}}{r^{3}}
\end{aligned}
$$

where $p^{\prime}=p-p_{\infty}, r_{i}$ is the vector from surface to observer location, $r=\left|r_{i}\right|$, and $n_{i}$ is the surface normal vector. Subscript $\infty$ denotes the ambient conditions. Farfield pressure is calculated in terms of the Fourier transform of nearfield sources $F_{1}$ and $F_{2}$ on $S$ using the following relation:

$$
\hat{p}(\mathbf{x}, f)=\int_{S}\left(\frac{i f}{2} \hat{F}_{1}(\mathbf{y}, f)+\frac{1}{4 \pi} \hat{F}_{2}(\mathbf{y}, f)\right) e^{-i 2 \pi r f / c_{\infty}} d \mathbf{y}
$$

where ${ }^{\wedge}$ indicates Fourier transformed quantities and $f$ is the frequency. It should be noted that Eq. 4 is derived based on the following premise:

1. Sound generated by flow outside surface $S$ is neglected.

2. Wave propagation outside surface $S$ is assumed to be linear.

3 . Refraction, attenuation and convection of sound waves outside surface $S$ is neglected.

\section{Simulation of Flow and Noise for Supersonic Jets}

The computation model were designed to mimic as close as possible the unheated (heated) jet B118 (B122) tested at UTRC's acoustic research tunnel (ART) facility. The same convergingdiverging (CD) nozzle geometry was chosen for the computations to match the experimental operating condition, which is exit Mach number of $M_{j}=1.5$ and jet exit static to chamber static temperature ratio is $T_{r}=1.0\left(T_{r}=1.743\right)$. The jet exhausts into an anechoic chamber which is subject to a wind tunnel flow with Mach number of $M_{t}=0.1$. The Reynolds number for the unheated (heated) jet based on the jet exit velocity, jet diameter, and viscosity at the nozzle exit is $2 \times 10^{6}\left(1 \times 10^{6}\right)$. The ART jet is surrounded by a forward flight open jet wind tunnel which exhausts into an anechoic chamber. The open jet velocity was set to a Mach number of $M_{t}=0.1$ to avoid overheating the anechoic chamber. The same $M_{t}=0.1$ free stream flow condition was used in the computational simulation.

The 3" diameter CD nozzle was designed using a methodof-characteristics to provide ideal expansion or shock free flow at the nozzle exit. A CD nozzle geometry was chosen for the baseline supersonic cases to facilitate the LES prediction of organized structure noise and fine scale mixing noise without the presence of shock cells. Future LES studies will address shock generated noise.

Baseline mean flow data was acquired to document the potential core length and sonic point in the exhaust stream. Radial profiles of total pressure, total temperature, and static pressure were acquired using a multi-probe from which Mach number, static temperature, and velocity were calculated. Surveys were conducted in the horizontal and vertical direction for the round nozzle to confirm that the traverse was aligned with the jet centerline. Centerline decay measurements along the jet axis were also acquired.

The computational domain is shown in figure 3. As shown, part of the nozzle geometry is included in the simulation domain. A constant plug-flow is applied to the inlet of the nozzle such that 
the desired Mach number and the temperature ratio are achieved at the nozzle lip. It should be noted that we "assume" that the flow issued from the nozzle is laminar ${ }^{1}$. Consequently, the grid resolution inside the nozzle is only adequate for a laminar flow. A slight coflow is applied to the jet surroundings to simulate the wind tunnel Mach number of $M_{t}=0.1$.

As shown in figure 3, a sponge layer is applied at the outlet of computational domain by switching the numerical operators to low-order dissipative discretization. By using this method, the turbulent structures and sound waves will be damped before approaching the outlet boundary.

The acoustic projection surface described in $\S 3$ is also shown in figure 3. To avoid the spurious noise caused by passage of flow structures through the end cap, the method introduced by Shur et al. (2005a) is applied; 15 end-caps spanning from $x=$ $20 D$ to $x=30 D$ end-cap are used to eliminate the uncorrelated (erroneous) sound. A sensitivity study of computed sound to the size of the acoustic projection surface and number of end-caps was carried out; similar conclusions to studies of Mendez et al. (2009) and Shur et al. (2005a) were obtained.

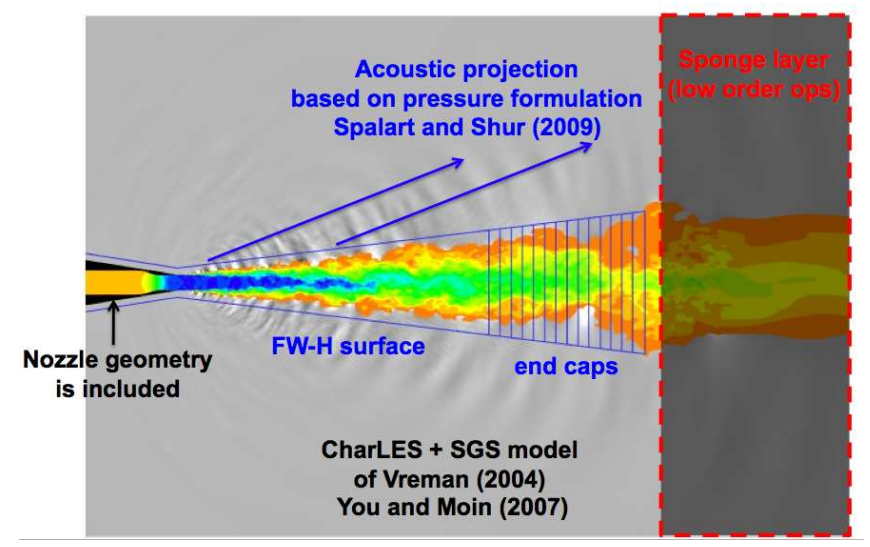

Figure 3. LES computational domain. Axial velocity field is shown in color, pressure is shown in grayscale.

Figure 4 demonstrates two sets of LES prediction stations corresponding to UTRCs experimental set up in which near field and far field microphones were used as reported by Schlinker et al. (2008). The near field stations, annotated as $P_{1}-P_{8}$, are based on the UTRCs detection of instability wave generated large-scale turbulence structures in the jet shear layer, generally accepted to be the source of aft-angle noise. The sensors are located in the hydrodynamic near field of the jet shear layer. The jet hydrodynamic near field has been demonstrated by UTRC to be the sound "source" containing the traveling wave pressure

\footnotetext{
${ }^{1}$ In the experiment, it is not known wether the flow issued immediately after nozzle is laminar or turbulent.
}

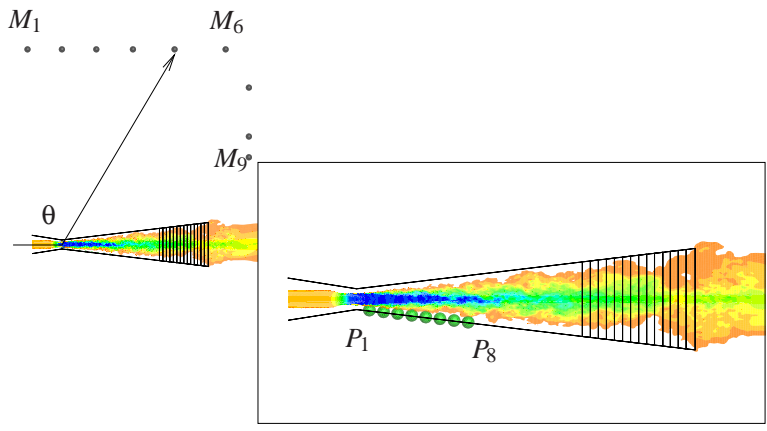

Figure 4. FWH surface and two sets of microphones used for calculation of noise; $P_{1}-P_{8}$ are nearfield pressure probes; $M_{1}-M_{9}$ are farfield microphones.

signature responsible for noise radiating to the far field in the aft direction. This was confirmed by Reba et al. (2009) using the measured instability wave packets and projecting them to the far field microphone stations, $M_{1}-M_{9}$.

The nearfield noise at each prediction station $\left(P_{1}-P_{9}\right)$ in figure 4 is calculated by recording the pressure signal from simulation on 48 equally-spaced azimuthal points. The sound spectrum is the average of spectra obtained from these azimuthal points. Farfield sound, at $M_{1}-M_{8}$, is calculated and averaged for 120 equally spaced azimuthal points. At every point, a sound spectrum is calculated according to Eq. 4 and the acoustic projection method described in $\S$.

For the unheated case corresponding to B118 jet two LES calculations were performed. These simulations are referred to as $118 \mathrm{C}$ and $118 \mathrm{M}$, where $\mathrm{C}$, and $\mathrm{M}$ stand for coarse and medium, respectively (the fine calculation are being carried out but not reported in this paper). The mesh for case $118 \mathrm{M}$ is shown in Figure 1; the resolution of $118 \mathrm{C}$ is identical to that of $118 \mathrm{M}$ except that the $118 \mathrm{C}$ does not have local refinement levels of 256 and 512 in the vicinity of the nozzle lip. The number of azimuthal grid points is 128 in these zones. Total number of computational cells for $118 \mathrm{C}$, and $118 \mathrm{M}$ are 11 million and 13 million, respectively. It should be noted that had we applied the resolution of 512 globally (instead of locally) the number of computational cells would be as large as 44 million. The azimuthal refinement from $118 \mathrm{C}$ to $118 \mathrm{M}$ was performed in order to study the effect of azimuthal resolution on the early transition of shear layers. In the previous study of Mendez et al. (2010), a maximum azimuthal resolution of 128 was applied in the entire domain. This resolution was far less than the axial or radial resolution in the early stages of shear layer development.

For the heated case corresponding to B122 only one simulation was conducted (called 122M hereafter) with the mesh being identical to that of case $118 \mathrm{M}$. Simulation parameters for all the computations are listed in table 1.

The results of LES computations are compared to: 


\begin{tabular}{c|cccc} 
& $M_{j}$ & $T_{r}$ & mesh size & $\Delta t c_{\infty} / D$ \\
\hline \hline $118 \mathrm{C}$ & 1.5 & 1.0 & $11 \mathrm{~m}$ & $2.0 \times 10^{-4}$ \\
$118 \mathrm{M}$ & 1.5 & 1.0 & $13 \mathrm{~m}$ & $2.0 \times 10^{-4}$ \\
$122 \mathrm{M}$ & 1.5 & 1.743 & $13 \mathrm{~m}$ & $1.0 \times 10^{-4}$
\end{tabular}

Table 1. Simulation parameters of LES computations.

1. Experiment carried out by Bridges \& Wernet (2008). Operating conditions for unheated (heated) jets in this experiment are $M_{j}=1.4$ and $T_{r}=1.0\left(T_{r}=1.765\right)$. These conditions are similar (but not exactly the same) to UTRC's experiment. Another difference is that, the velocity of coflow in UTRC's experiment is $M_{t}=0.1$, which is larger than $M_{t}=0.008$ used by Bridges \& Wernet (2008).

2. Empirical correlation of Witze (1974), in which a relation is given for decay of axial velocity along the centerline of the jet.

3. A "tuned" RANS simulation. A two-dimensional axisymmetric RANS simulation for the unheated jet was performed. The spreading rate of the shear layer and size of the potential core obtained from RANS was used as a guideline for designing the LES mesh. We employed the $k-\varepsilon$ model with modifications of Tam \& Ganesan (2004) for supersonic hot jets. Axial mean velocity obtained from this calculation is demonstrated in figure 5. The results show excellent agreement with experimental correlations of Witze (1974).

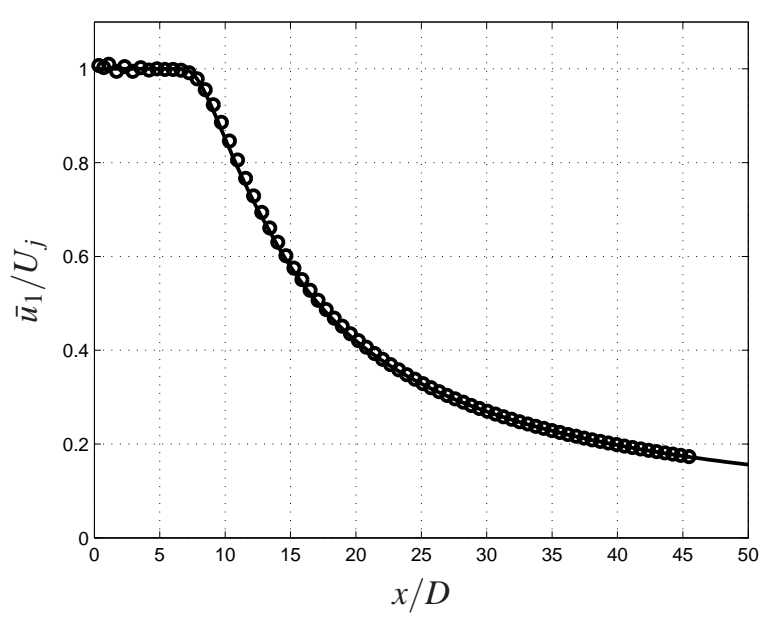

Figure 5. Centerline axial velocity from, o RANS simulation; - experimental correlation of Witze (1974) for unheated jet.

4. UTRC measurements (blind comparisons).
The data from the first three items are only used for comparison to first- and second-order statistics of axial velocity while a comparison of velocity, near-filed and far-field are conducted for the last item. In the following, we first qualitatively describe the flow, then present a mesh resolution study by examining the axial velocity of the unheated jet in the vicinity of nozzle. At last, flow and noise issued by both unheated and heated jets will be compared to UTRC's measurements.

\subsection{Visualization and qualitative description of the flow}

Instantaneous temperature fields for both unheated and heated jet calculation as well as the dilatation (divergence of velocity) field for the unheated jet are shown in figures 6,7 , and 8 , respectively. In these figures, the computational mesh is partially shown in planes normal to the axis of the jet. Figures 6 and 7 demonstrate the mixing of heated flow with the colder flow. According to these figure, zonal refinement of the mesh is required for capturing small scales of the flow in the vicinity of the nozzle lip. These small scales are caused by the transition of thin shear layer to turbulence.

According to temperature fields presented(i.e. figures 6 and 7 ), the thin shear layer issued from the nozzle is laminar at the early stages (see plane-cut at $x=0.015 D$ ). Numerical effects (see plane-cut at $x=0.5 D$ ) trigger the transition to turbulence at about a diameter downstream of the nozzle. The turbulent flow mixes rapidly at the farther stages downstream of the nozzle. Mach wave radiation of sound initiated from the onset of mixing layer is hardly visible in the temperature field. It is, however, clearly visualized through the dilatation field in figure 8. Similar to laboratory experiments, exact pressure matched condition cannot be achieved in simulation environment. Consequently, residual shock cells are formed in the vicinity of the nozzle. The shock cell structure is visible from the contour plots of averaged density in figure 9.The presence of the cells suggests the nozzle is operating severely off design, but, the average density parameter has accentuated the shock strength. Invariance in the shock deflection angles downstream of each centerline intersection indicates the shocks are actually weak. The shock angles retain the same geometry over multiple cells. The shocks were minimized in the experimental studies by varying the nozzle pressure ratio over a small range centered at the design value and determining when the minimum sound level was observed at the 90 degrees microphone station in figure 4 . This corresponded to $M=1.495$ for the unheated condition which was considered to be the shock free operating condition.

\subsection{Mean flow and mesh resolution study}

After simulations reached statistical convergence, flow statistics were collected for 146, 168, and 189 non-dimentional time units for $118 \mathrm{C}, 118 \mathrm{M}$, and $122 \mathrm{M}$, respectively. Time is 


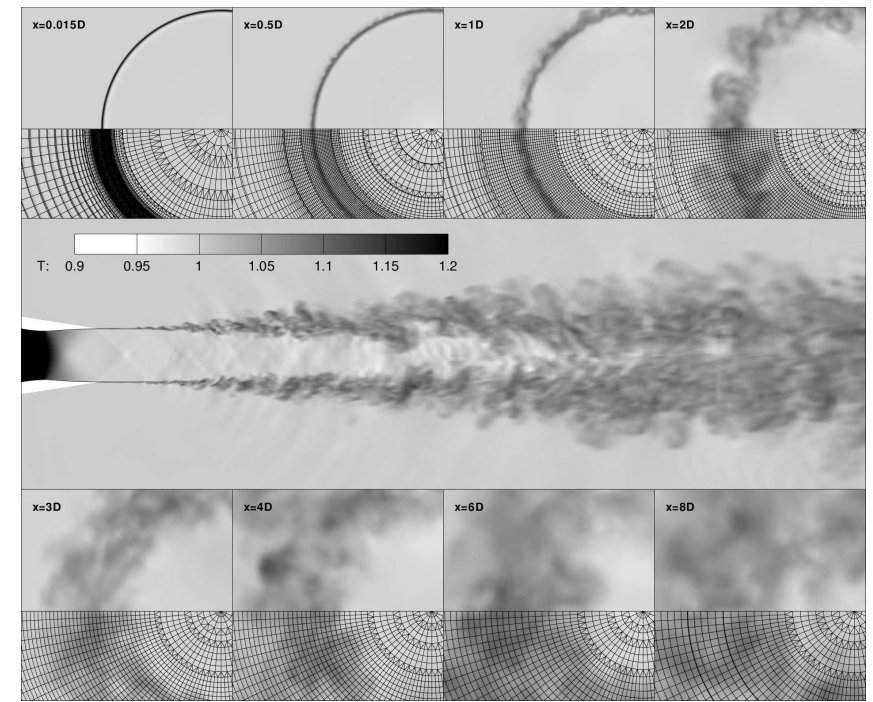

Figure 6. Temperature field for unheated jet (simulation 118M).

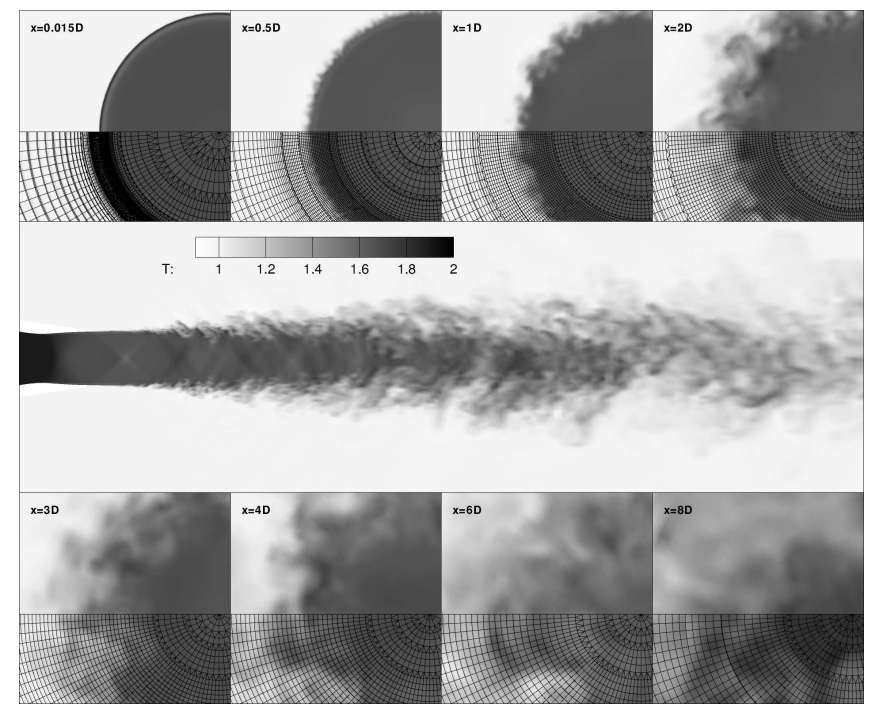

Figure 7. Temperature field for heated jet (simulation 122M).

non-dimensionalized by centerline jet velocity and jet diameter at nozzle exit. First- and second-moment statistics presented here are averaged in both time and azimuthal direction. Figures 10(a) and 11(a) demonstrate the mean and r.m.s. of the axial velocity on the centerline of the jet for unheated and heated cases. For azimuthally-refined calculations, the length of the potential core is approximately 1.5 diameter shorter than experiments carried out by Bridges \& Wernet (2008). This discrepancy is larger compared to UTRC measurements, in which the size of potential core is about 2 diameters longer than the $118 \mathrm{M}$. An increase is expected since the Mach number of the UTRC jet is higher than the condition reported by Bridges \& Wernet (2008). The r.m.s. velocity profile has a similar shape and magnitude to ex-

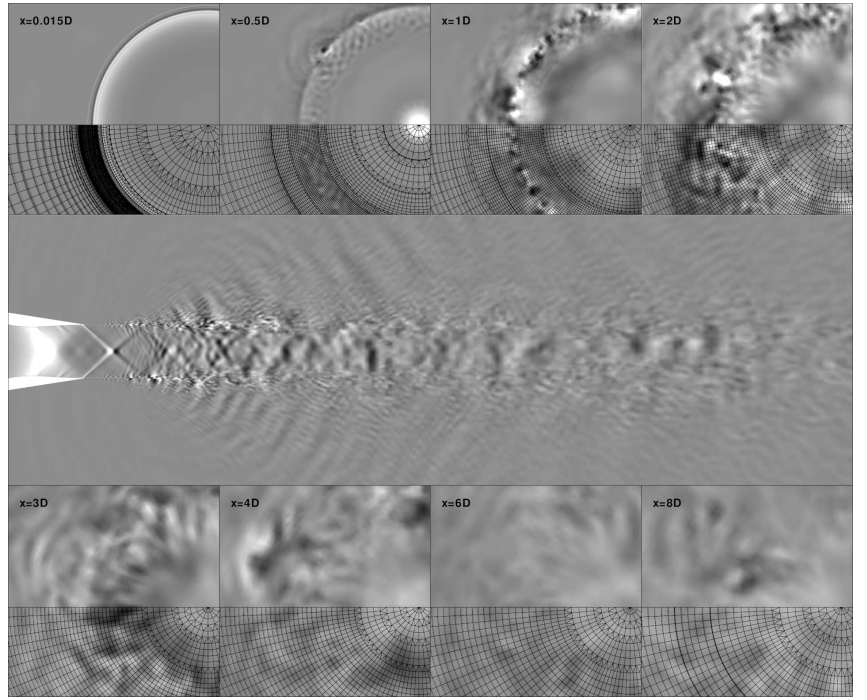

Figure 8. Dilatation field for unheated jet (simulation 118M).

perimental measurements for both heated and unheated cases. It is, however, shifted to upstream due to the existence of a shorter potential core.

Figures 10(b) and 11(b) show the axial mean and r.m.s. velocity along the lipline; shape and magnitude of both mean and r.m.s. profiled are in reasonable agreement with experiments for both jet conditions for $x>5 D$. However, LES predicts a peak in r.m.s. values approximately 1.5 diameter downstream of the nozzle. This peak, which is not present in the experiment, is caused by laminar-to-turbulence transition of mixing layers. A similar trend is observed in the radial profiles of axial velocity (see figures 12 and 13). The r.m.s. values are over-predicted by LES in early stages of the development of mixing layers. The agreement improves significantly at downstream stations.

According to these results, increasing the azimuthal resolution in the vicinity of the nozzle significantly affects the transition of the mixing layer. The effect of azimuthal resolution on transition is described next.

Mean and r.m.s. of axial velocity along the centerline of the jet are plotted in figure 10 (a). The shear layer in coarse LES (118C) spreads faster, and a shorter potential core is, therefore, predicted. The potential core length as well as r.m.s. values of axial velocity predicted by the azimuthally-refined LES (118M) is closer to the measurements of Bridges \& Wernet (2008) as well as UTRC results. Mean and r.m.s of axial velocity along the lip-line of the jet is plotted in figure 10 (b). Mean velocities are very similar; however, azimuthally-refined LES (118M) predicts an earlier transition with lower r.m.s. values which is closer to the experiments of Bridges \& Wernet (2008). Radial profiles of axial velocity shown in figure 12 also demonstrate that the azimuthally-refined LES is in better agreement with the experiment. According to figure 12, the difference between 118C 


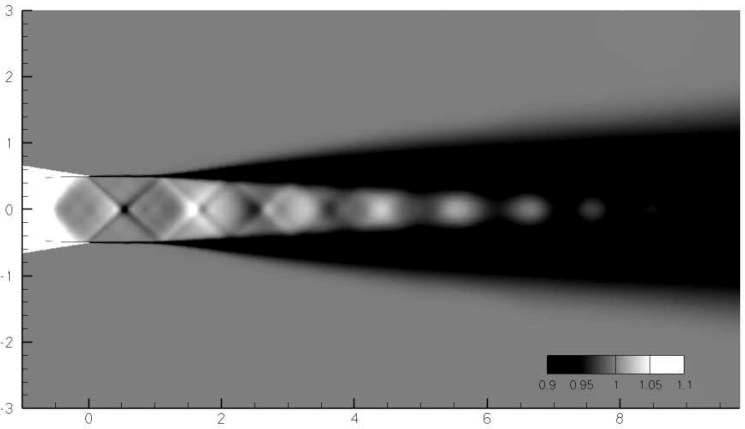

(a) $\rho / \rho_{\infty}$ for unheated jet

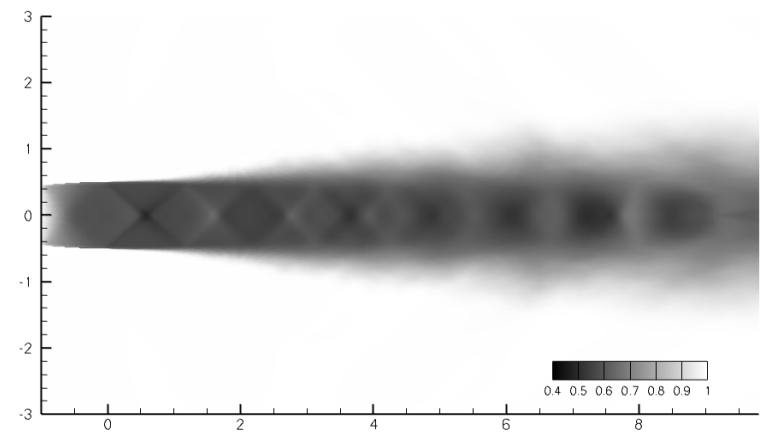

(b) $\rho / \rho_{\infty}$ for heated jet

Figure 9. Shock cells visualized by averaged density field,

and $118 \mathrm{M}$ is more pronounced in the early stages of transition of mixing layers to turbulence. Furthermore, the level of velocity fluctuation is higher in the transition predicted by $118 \mathrm{C}$.

According to above observations, LES with azimuthal refinement $(118 \mathrm{M})$ predicts an earlier transition of laminar shear layer to turbulence than LES without the refinement (118C). This earlier transition is likely due to instability modes with higher azimuthal wave-numbers, which are supported by the refined grid.

\section{UTRC experiment and blind comparisons}

The results of medium-size simulations (118M and 122M) were sent to UTRC for blind comparisons. Mean axial velocity in the jet plume, nearfield noise, and farfield noise are compared.

\subsection{Mean flow}

Figures 14 and 15 show the comparison of axial velocity for unheated and heated jets, respectively. The major discrepancy between LES and measurement is the size of potential core; the length of potential core is under-predicted by LES. Accordingly, the radial profiles before $x / D=7$ are in good agreement (see figures 14(a), 14(b), and 15(a)); while for stations further downstream, maximum velocity is under-predicted. One possible reason for the under-prediction of the length of potential core

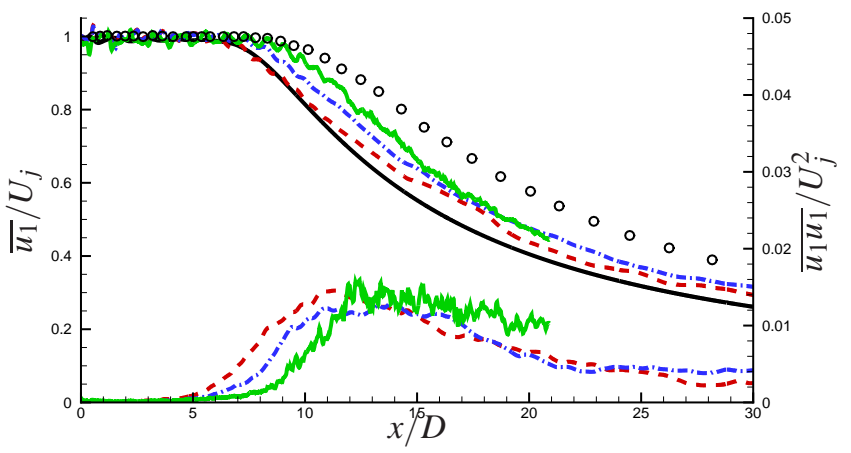

(a) centerline

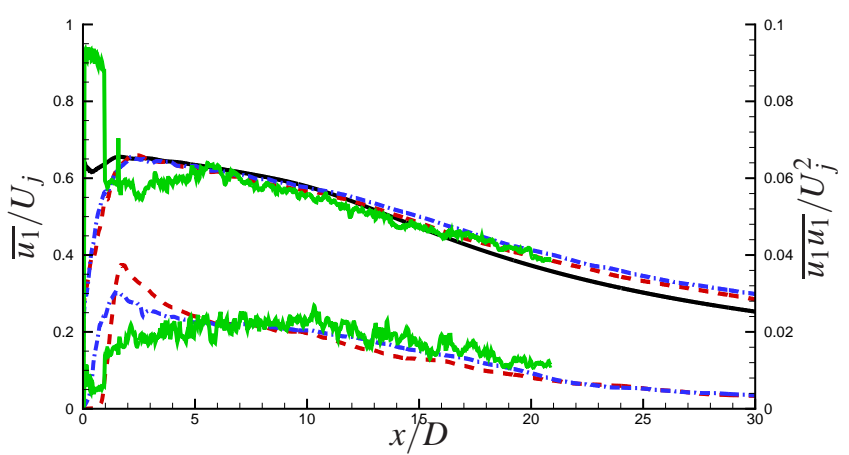

(b) lipline

Figure 10. Comparison of mean and r.m.s. axial velocity for unheated jet; — , RANS; ---- , LES(118M); - - - LES(118C); — , measurement of Bridges \& Wernet (2008) for $M_{j}=1.4$; o , UTRC measurements for $M_{j}=1.5$.

is the lack of resolution in the jet plume; as shown in figure 10, the length of potential core was increased by resolving the mesh from $118 \mathrm{C}$ to $118 \mathrm{M}$.

\subsection{Nearfield sound}

Figures 16 and 17 show the comparison of sound spectra for unheated and heated jets, respectively. Nearfield probe stations at which the comparison are performed are shown in figure 4 . LES digitized data was processed using the same scheme as used in the experiment digital data to allow direct comparison of spectra. The overall agreement in terms of the shape of spectra and levels is very good. Higher levels of pressure at high frequencies predicted by LES at probes farther downstream indicates the lack of resolution at these regions; the computational grid cannot support small structures at such high frequencies. As a result, the energy associated with these structures appear as numerical noise and contaminate the solution at higher frequencies. Similar to comparison of mean axial velocity, grid refinement from $118 \mathrm{M}$ to $118 \mathrm{~F}$ (where $\mathrm{F}$ stands for a Fine grid) will likely resolve this issue. Fine grid calculations will be reported in the 


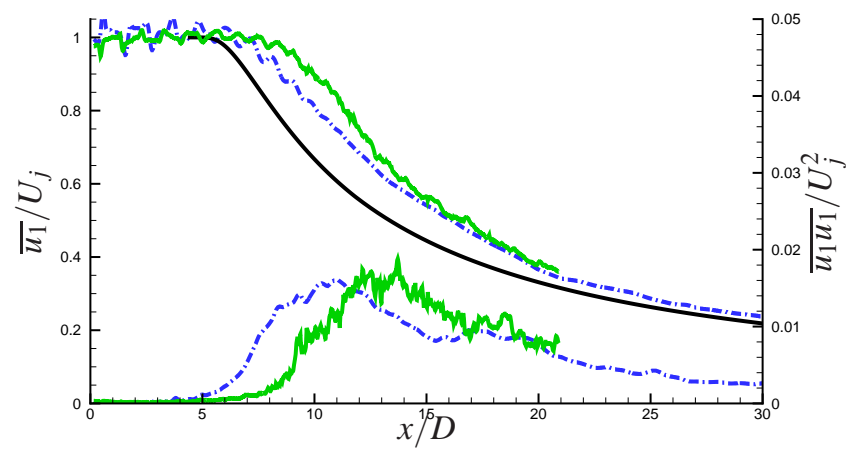

(a) centerline

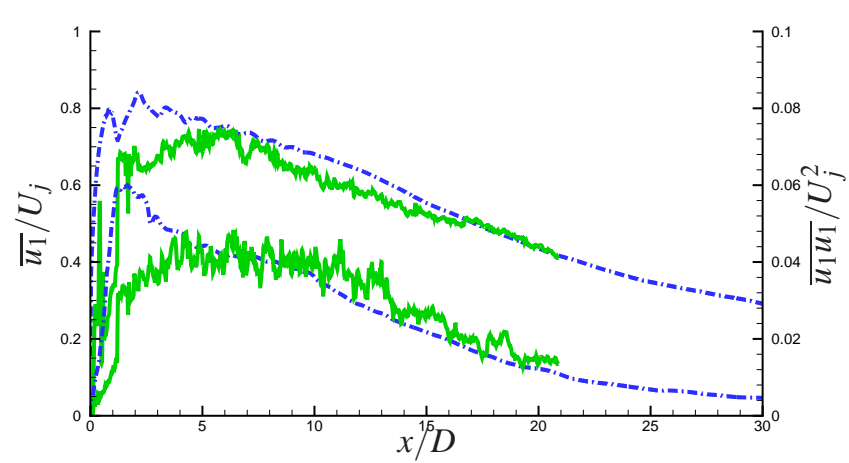

(b) lipline

Figure 11. Comparison of mean and r.m.s. axial velocity for heated jet; — , empirical correlations according to Witze (1974); ---- , LES(122M); - , measurement of Bridges \& Wernet (2008) for $M_{j}=$ 1.4 and $T_{r}=1.76$.

future. The low frequency peaks at $M_{1}$ that appear in both LES and the UTRC experiment is hypothesized to be vortex pairing in early stages of shear layer mixing. The source of high frequency noise in experimentally measured spectra at station $M_{1}$ (see figures 16(a) and 17(a)) is hypothesized to be the weak Mach wave radiation propagating to aft angles in the dilation field shown in figure 8 and is being investigated further.

\subsection{Farfield sound}

Figure 18 shows comparisons of the farfield sound for the unheated jet as a function of the microphone stations in figure 4 while figure 19 shows the comparison for the heated jet. Similar to the nearfield comparisons, the LES far field data processing emulated the experimental data processing. The narrowband spectra span the range of dominant jet noise directivity angles in the aft quadrant; from the most aft measurement angle in figure 4 corresponding to $M_{9} / 155^{\circ}$ to microphone $M_{5} / 120^{\circ}$.

According to these figures, the agreement is reasonable for these aft angles, in particular for microphone $M_{8}$ at $\theta=150^{\circ}$. However, sound levels are over predicted as the microphone sta-
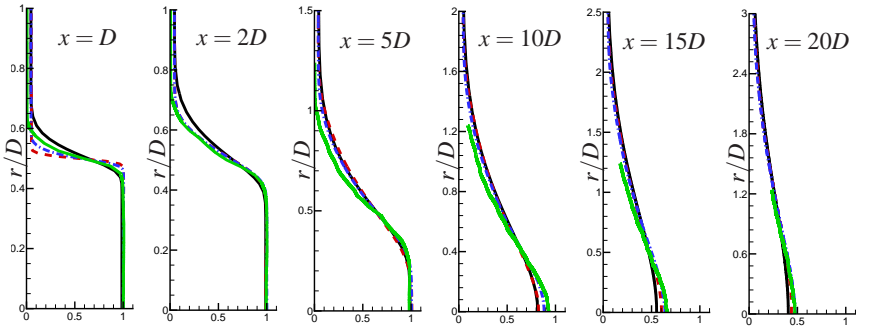

(a) $\bar{u}_{1} / U_{j}$
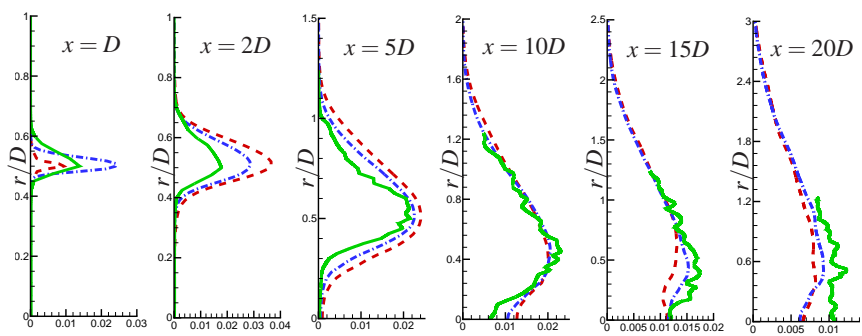

(b) $\overline{u_{1} u_{1}} / U_{j}^{2}$

Figure 12. Comparison of mean and r.m.s. axial velocity;

RANS; - - - - LES(118M); - - - , LES(118C); —— , measurement of Bridges \& Wernet (2008) for $M_{j}=1.4$.

tions approach lower inlet angles and high frequencies. This become more pronounced for microphones straddling the $90^{\circ}$ station in figure $4\left(M_{4} / 110^{\circ}\right.$ to $\left.M_{1} / 80^{\circ}\right)$. This over prediction is in agreement with the fact that the mesh is under-resolved in the jet plume and in the vicinity of potential core closure point; high frequency noise at small inlet angles is connected to turbulent mixing noise originating in these regions. Again, grid refinement in these regions is likely to improve the results. Another factor that can contribute to this difference is the viscous attenuation and background convection of sound waves which are present in the experiment and not modeled in the acoustic projection method. Based on the results and discussions presented above, to improve the quality of far field sound comparison:

1. A fine calculation $(118 \mathrm{~F})$ is being integrated further to obtain converged statistics for noise computation,

2. The experimental experimental spectra are being converted to a "lossless atmosphere" to remove viscous attenuation effects

3. The background convection in the shear layer is being added to the acoustic projection method by utilizing Greens function of a convective wave equation.

\section{Summary and future directions}

In this work, we used unstructured LES for prediction of flow and noise issued from supersonic jets. The flow is resolved using a finite volume method in the region of sound generation; 

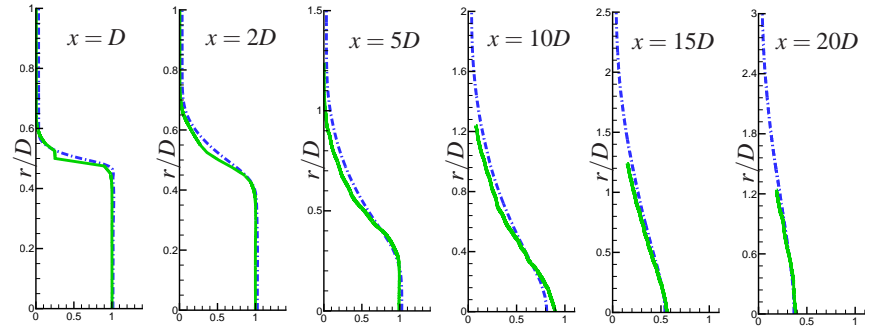

(a) $\bar{u}_{1} / U_{j}$
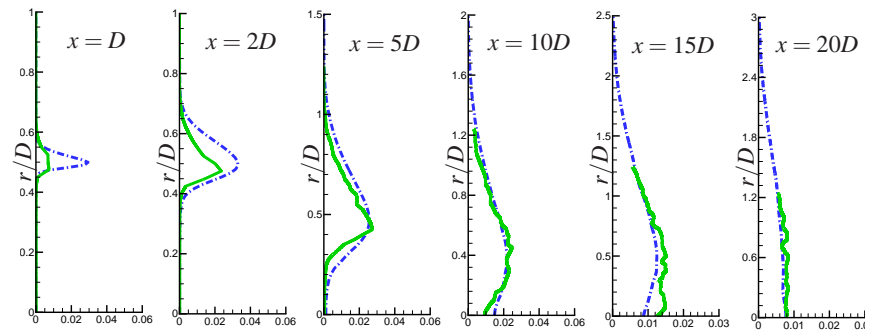

(b) $\overline{u_{1} u_{1}} / U_{j}^{2}$

Figure 13. Comparison of mean and r.m.s. axial velocity; ---- , LES(122M); - , measurement of Bridges \& Wernet (2008) for $M_{j}=$ 1.4 and $T_{r}=1.76$.

then an acoustic projection method is applied for computation of farfield noise. The finite volume method is designed such that it introduces dissipation only in the regions of mesh where the quality of grid is less than ideal; this dissipation is necessary to avoid numerical instabilities. Using this numerical scheme allowed us to use high quality meshes with aggressive local grid refinement.

Using the LES framework, we simulated an isothermal and a hot jet; the nozzle geometry and operating conditions matched those of jets previously tested at UTRC's ART facility. For the isothermal case we carried out a local mesh refinement study where we provided higher azimuthal resolution close to the nozzle lips. While the additional resolution did not significantly increase our computational cost, the quality of solution in the vicinity of nozzle improved significantly.

While refinement of the current LES based simulation methodology is continuing, the results are promising for exploiting high fidelity methods to understand supersonic jet noise mechanisms and their control. Comparisons between LES predicted near field hydrodynamic pressure fluctuations and UTRC measured data show good agreement for the instability generated organized turbulence structures/wave packet spectra. This observation applies for both unheated and heated jets at the $M=1.5$ conditions representative of tactical aircraft. Far field acoustic spectrum comparisons also show reasonable agreement at aft directivity angles where noise levels dominate by $20 d B$ over observer stations at 90 degrees to the jet centerline. For observer angles near 90 degrees and high frequencies predicted versus measured spectrum differences occur due to the computational mesh being under-resolved in the jet plume and in the vicinity of potential core closure point; high frequency noise at small inlet angles are connected to turbulent mixing noise originating in these regions. Again, grid refinement in these regions is likely to improve the results.

\section{Acknowledgement}

We acknowledge Dr. Shoreh A. Hajiloo from CASCADE Technologies Inc. for her contribution in designing the computational mesh, RANS simulations, and reviewing the present paper. We are also thankful to Mr. Mohammad Shoeybi from Stanford University for many good discussions and his help with the acoustic projection module.
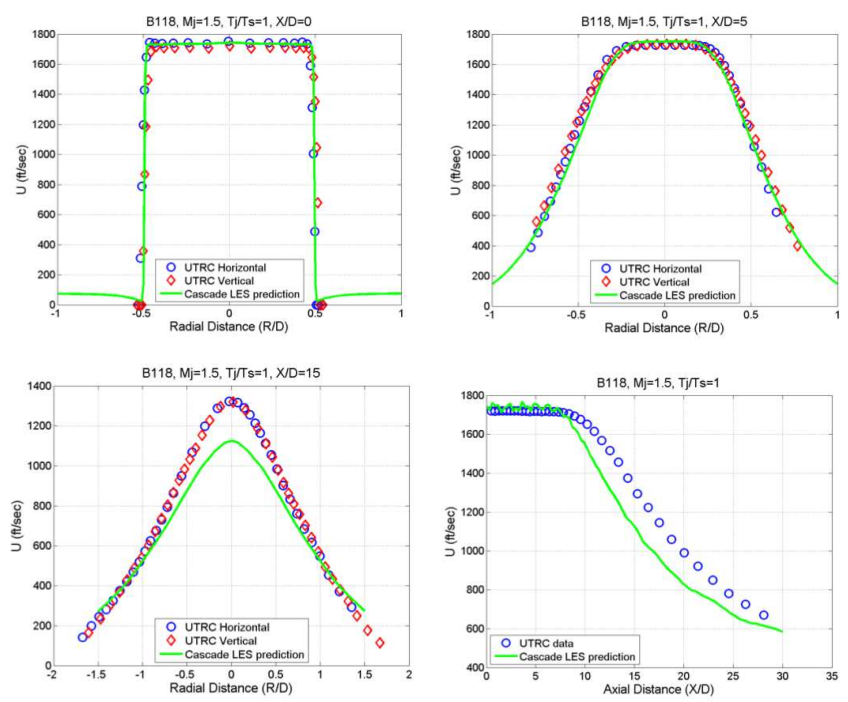

Figure 14. Comparison of streamwise mean velocity for B118
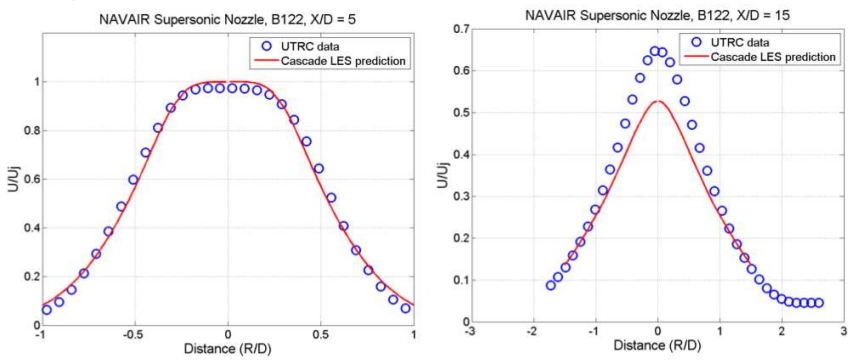

Figure 15. Comparison of streamwise mean velocity for B122 

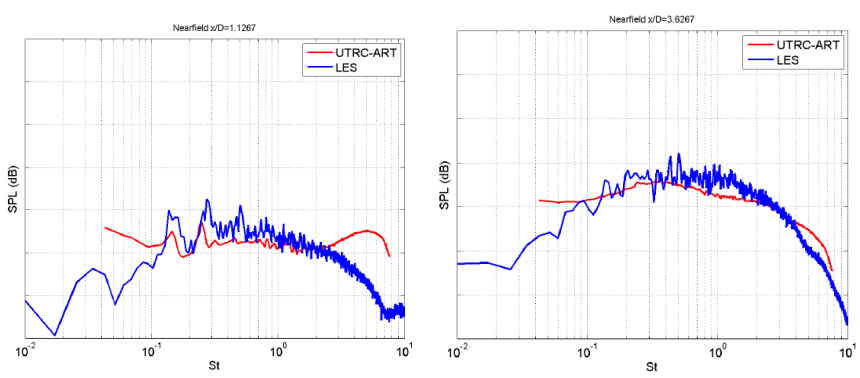

(a) $P_{1}$

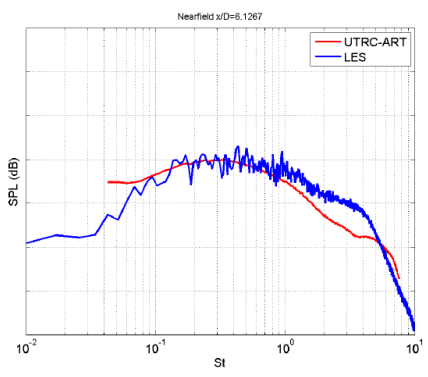

(c) $P_{5}$ (b) $P_{3}$

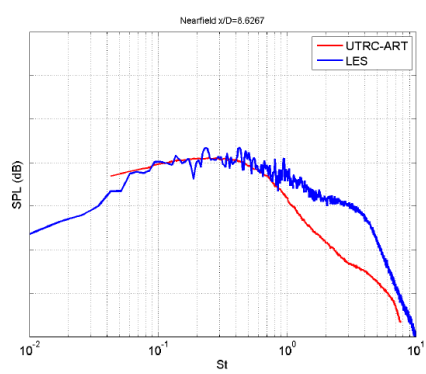

(d) $P_{7}$

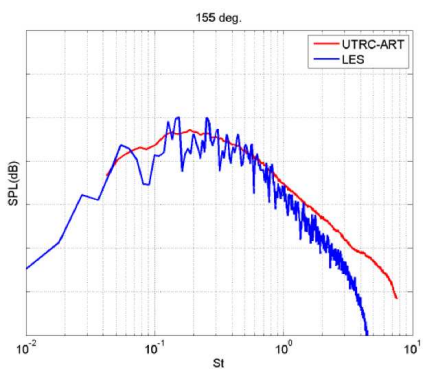

(a) $M_{9}$

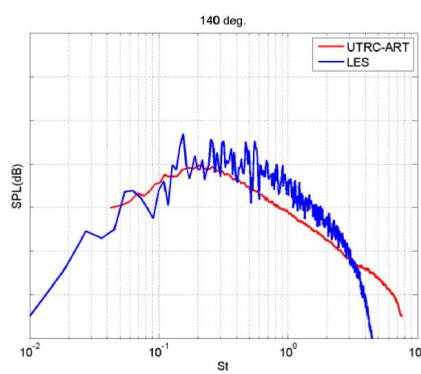

(c) $M_{7}$

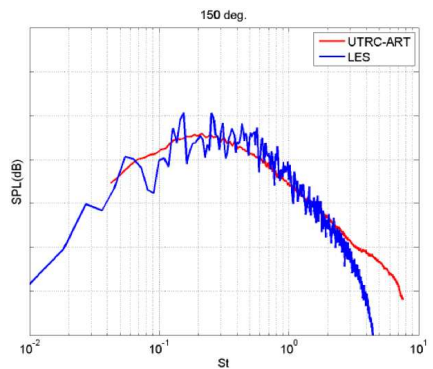

(b) $M_{8}$

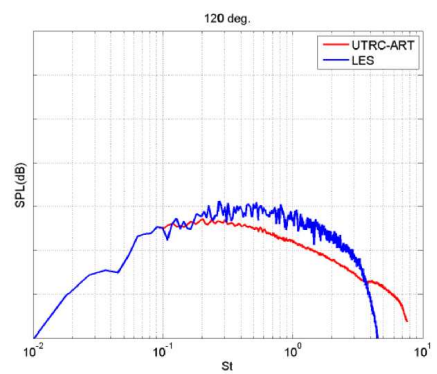

(d) $M_{5}$

Figure 16. Comparison of nearfield sound for B118.- Experiment;- LES

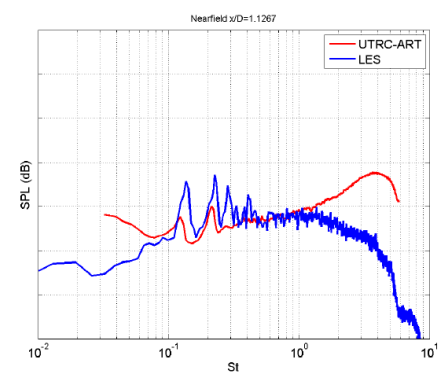

(a) $P_{1}$

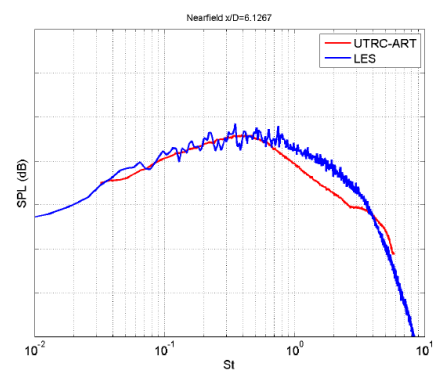

(c) $P_{5}$

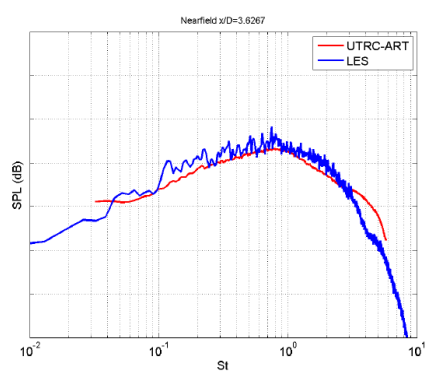

(b) $P_{3}$

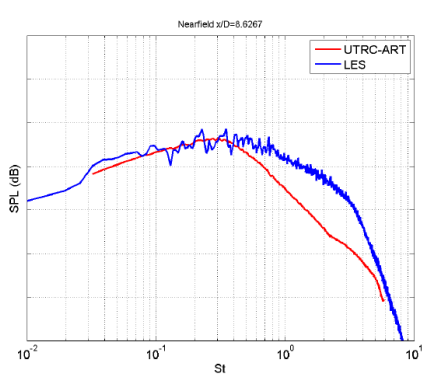

(d) $P_{7}$

Figure 17. Comparison of nearfield sound for B122.___Experiment;- LES

Figure 18. Comparison of farfield sound for B118.- Experiment; - LES
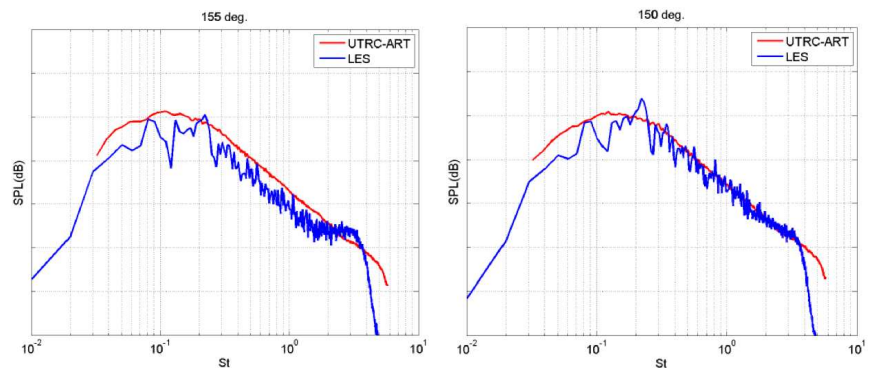

(a) $M_{9}$

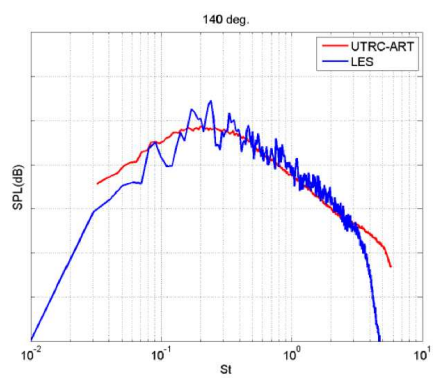

(b) $M_{8}$

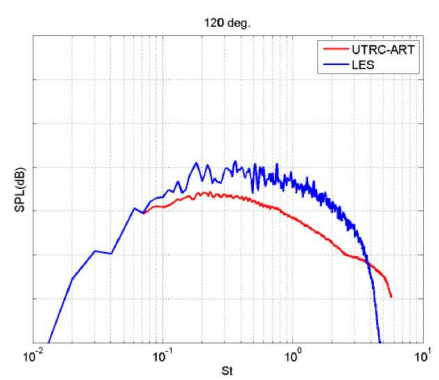

(c) $M_{7}$

(d) $M_{5}$

Figure 19. Comparison of farfield sound for B122._- Experiment; - LES 


\section{REFERENCES}

BODONY, D. 2005 Analysis of sponge zones for computational fluid mechanics. Journal of Computational Physics 212, 681702.

Bodony, D. J. \& Lele, S. K. 2008 Current status of jet noise predictions using large-eddy simulation. AIAA J. 46, 346-380.

BRIDGeS, J. \& WERnET, M. P. 2008 Turbulence associated with broadband shock noise in hot jets. In 14th AIAA/CEAS Aeroacoustics Conference, Vancouver, CANADA, 5-7 May 2008.

Ffowcs Williams, J. E. \& Hawkings, D. L. 1969 Sound Generation by Turbulence and Surfaces in Arbitrary Motion. Royal Society of London Philosophical Transactions Series A 264, 321-342.

Germano, M., Piomelli, U., Moin, P. \& CABot., W. 1991 A dynamic subgrid-scale eddy viscosity model. Phys. of Fluids 3 (7), 1760-1765.

Ghosal, S., Lund, T., Moin, P. \& Akselvoll, K. 1995 A dynamic localization model for large-eddy simulation of turbulent flows. Journal of Fluid Mechanics 286, 229-255.

Ham, F., Mattsson, K., Iaccarino, G. \& Moin, P. 2007 Towards Time-Stable and Accurate LES on Unstructured Grids, pp. 235-249. springer, vol. 56 of Lecture Notes in Computational Science and Engineering.

Harten, A., LAX, P. \& LeER, B. V. 1983 On upstream differencing and godunov-type schemes for hyperbolic conservation laws. SIAM review 15, 35-61.

Hill, D. J. \& Pullin, D. I. 2004 Hybrid tuned centerdifference-weno method for large eddy simulations in the presence of strong shocks. Journal of Computational Physics 194 (2), 435-450.

Khalighi, Y., Mani, A., Ham, F. \& Moin, P. 2010 Prediction of sound generated by complex flows at low mach numbers. AIAA J. 68 (2), 306-316.

Ladeinde, F., Cai, X., Alabi, K., Reba, R., Schlinker, R. H. \& SimONiCH, J. 2008 On the connection between nearfield and far-field solutions of high-speed jet noise. In AIAA Aerospace Science Meeting. Reno, AIAA Paper, AIAA-200811.

LILLY, D. K. 1992 A proposed modification of the Germano subgrid-scale closure method. Phys. Fluids A 4 (3), 633-35.

Lo, S. C., Blaisdell, G. A. \& Lyrintzis, A. S. 2008 Numerical simulation of supersonic jet flows and their noise. In AIAA Aerospace Science Meeting. Reno, AIAA Paper, AIAA2008-2970.

LYRINTZIS, A. S. 2003 Surface integral method in computational aeroacoustics- From the CFD near-field to the Acoustic far-field. International Journal of Aeroacoustics 2 (2), 95-128.

Mani, A., LARsson, J. \& MoIn, P. 2009 Suitability of artificial bulk viscosity for large-eddy simulation of turbulent flows with shocks. Journal of Computational Physics 228, 73687374 .
Marsden, A. L., WAng, M., Dennis, J. E. \& Moin, P. 2007 Trailing edge noise reduction using derivative-free optimization and large-eddy simulation. Journal of Fluid Mechanics 572, 13-36.

Mendez, S., Shoeybi, M., Sharma, A., Ham, F. E., Lele, S. K. \& MoIn, P. 2010 Large-eddy simulations of perfectlyexpanded supersonic jets: Quality assessment and validation. In 48th AIAA Aerospace Sciences Meeting Including the New Horizons Forum and Aerospace Exposition. Orlando, Florida, AIAA paper 2010-271.

Mendez, S., Shoeybi, M., Sharma, A., Lele, S. K. \& MoIN, P. 2009 Post-processing of large-eddy simulations for jet noise predictions. Center for Turbulence Research Annual Research Briefs, Stanford, CA .

Mittal, R. \& Moin, P. 1997 Suitability of upwind-biased finite difference schemes for large-eddy simulation of turbulent flows. AIAA Journal .

Moin, P., Squires, K., CABot, W. \& Lee, S. 1991 A dynamic subgrid-scale model for compressible turbulence and scalar transport. Phys. Fluids A 3-11, 2746-57.

RebA, R. A., Simonich, J. C. \& Schlinker, R. H. 2008 Decomposition of high speed jet noise: Source characteristics and propagation effects. In 14th CEAS/AIAA Aeroacoustics Conference. Vancouver, British Columbia, AIAA paper 2008-2891.

RebA, R. A., Simonich, J. C. \& Schlinker, R. H. 2009 Sound radiated by large-scale wave-packets in subsonic and supersonic jets. In 15th CEAS/AIAA Aeroacoustics Conference. Miami, Florida, AIAA paper 2009-3256.

Schlinker, R. H., Simonich, J. C., Reba, R. A., ColoNIUS, T. \& LADEINDE, F. 2008 Decomposition of high speed jet noise: Source characteristics and propagation effects. In 14th CEAS/AIAA Aeroacoustics Conference. Vancouver, British Columbia, AIAA paper 2008-2890.

SHI, J., HU, C. \& SHU, C. 2002 A technique of treating negative weights in weno schemes. Journal of Computational Physics 175, 108-127.

Shoeybi, M., Svard, M., Ham, F. \& Moin, P. 2009 An adaptive hybrid implicit-explicit scheme for the DNS and LES of compressible flows on unstructured grids. submitted to Journal of Computational Physics .

Shur, M., Spalart, P., Strelets, M. \& Travin, A. 2003 Towards the prediction of noise from jet engines. International Journal of Heat and Fluid Flow 24, 551-561.

Shur, M. L., Spalart, P. R. \& Strelets, M. K. $2005 a$ Noise prediction for increasingly complex jets. part i: Methods and tests. International Journal of Aeroacoustics 4 (3-4), 213-246.

Shur, M. L., Spalart, P. R. \& Strelets, M. K. $2005 b$ Noise prediction for increasingly complex jets. part ii: Applications. International Journal of Aeroacoustics 4 (3-4), 247266. 
SPAlART, P. R. \& SHUR, M. L. 2009 Variants of Ffowes Williams-Hawkings equation and their coupling for simulation of hot jets. International Journal of Aeroacoustics 4 (3-4), 247-266.

Spalart, P. R., Shur, M. L. \& Strelets, M. K. 2007 Identification of sound sources in large-eddy simulations of jets. In 13th CEAS/AIAA Aeroacoustics Conference. Rome, Italy.

TAM, C. K. W. \& GAnESAn, A. 2004 Modified k-epsilon turbulence model for calculating hot jet mean flows and noise. AIAA J. 43, 26-34.

TUCKER, P. G. 2004 Novel miles computations for jet flows and noise. International Journal of Heat and Fluid Flow 25, 625635.

VREMAN, A. 2004 An eddy-viscosity subgrid-scale model for turbulent shear flow: Algebraic theory and applications. Physics of Fluids 16, 3570.

WAng, M., Freund, J. B. \& Lele, S. K. 2006 Computational prediction of flow-fenerated sound. Annu. Rev. Fluid Mech 38, 483-512.

WANG, M. \& MoIN, P. 2000 Computation of trailing edge flow and noise using large-eddy simulation. AIAA J. 38, 22012209.

WitZE, P. O. 1974 Centerline velocity decay of compressible free jets. AIAA J. 12, 417-418.

Xia, H., Tucker, P. G. \& Eastwood, S. 2009 Large-eddy simulations of chevron jet flows with noise predictions. International Journal of Heat and Fluid Flow 30, 1067-1079.

You, D. \& MoIN, P. 2007 A dynamic global-coefficient subgrid-scale eddy-viscosity model for large-eddy simulation in complex geometries. Physics of Fluids 19 (6), 065110. 